Max-Planck-Institut für demografische Forschung

Max Planck Institute for Demographic Research

Konrad-Zuse-Strasse 1 - D-18057 Rostock · GERMANY

Tel +49 (0) 3812081 - 0; Fax +49 (0) 3812081 - 202;

http://www.demogr.mpg.de

MPIDR WORKING PAPER WP 2005-018

AUGUST 2005

\title{
Aging: damage accumulation versus increasing mortality rate
}

Maxim S. Finkelstein (finkelm@sci.uovs.ac.za)

This working paper has been approved for release by: James W. Vaupel (jwv@ demogr.mpg.de) Head of the Laboratory of Survival and Longevity.

(C) Copyright is held by the authors.

Working papers of the Max Planck Institute for Demographic Research receive only limited review. Views or opinions expressed in working papers are attributable to the authors and do not necessarily reflect those of the Institute. 


\title{
AGING: DAMAGE ACCUMULATION \\ VERSUS INCREASING MORTALITY RATE
}

\author{
M.S. Finkelstein \\ Department of Mathematical Statistics \\ University of the Free State \\ PO Box 339, 9300 Bloemfontein, Republic of South Africa \\ (e-mail: FinkelM@sci.uovs.ac.za) \\ and \\ The Max Planck Institute for Demographic Research, \\ Rostock, Germany
}

\begin{abstract}
If aging is understood as some process of damage accumulation, it does not necessarily lead to increasing mortality rates. Within the framework of a suggested generalization of the Strehler-Mildwan (1960) model, we show that even for models with monotonically increasing degradation, the mortality rate can still decrease. The decline in vitality and functions, as manifestation of aging, is modeled by the monotonically decreasing quality of life function. Using this function, the initial lifetime random variable with ultimately decreasing mortality rate is 'weighted' to result in a new random variable which is already characterized by the increasing rate.
\end{abstract}

Keywords: Aging, mortality, accumulated damage, quality of life function

\section{INTRODUCTION}

Mortality rates of most species increase with time at least after the start of reproduction. For advanced ages they sometimes also tend to level off or even to decrease, which among other reasons, can be due to population heterogeneity (Vaupel et al, 1979). In this note, however, we will focus only on a homogeneous case. Does a pos- 
sible deceleration in mortality mean a deceleration in aging? This certainly depends on a definition of aging and we show under certain assumptions that when overall aging of an organism is understood as some accumulation of damage (additive degradation), and this is our assumption, it does not always lead to increasing mortality rates. Therefore, we distinguish between the deterioration per se and its manifestation in the form of increasing mortality rate which is likely but not always to occur. In this way we partially argue with Finch (1990), where he defines senescence as "age-related changes in an organism that adversely affect its vitality and functions, but most important increase the mortality rate..." (see also Vaupel et al (2004) for the corresponding discussion). We, on the contrary, emphasize the fact that accumulated damage of some kind, eventually defines these age related changes in an organism and, combined with other factors, determines the shape of the mortality rate. This approach is definitely not a new one, and was recently supported in a rather general setting by Aalen and Geising (2001) in their path breaking paper with a speaking for itself title: Understanding the shape of the hazard rate: a process point of view.

Consider an organism in a post-reproductive phase of life, when the accumulated damage already noticeably results in negative age related changes. On the other hand, assume that the mortality rate is leveling off or even decreasing at sufficiently advanced age, which is observed in humans and some other species. How can these, at first sight, contradictory properties coexist? Firstly, we show that under certain assumptions this can still be the case, and, secondly, we suggest how, at least formally, to deal with and to interpret the stated contradiction.

Apart from the shape of mortality rate, the following question can be asked: is the 'value' of a unit of a lifetime of humans at sufficiently advanced ages the same as at previous phases of life? Humans at advanced ages usually have restrictions of various kinds, showing a substantial decrease in vitality and functions and therefore in some sense decreasing a quality of life at this stage. It should be noted, however, that although formally vitality and functions of humans decrease at all adult ages, the noticeable decline in the corresponding quality of life due to these processes occurs usually only at relatively advanced ages.

In Section 2, using a generalization of the Strehler-Mildwan (1960) model, we show that even for models with monotonically increasing degradation, the mortality rate can still decrease. In Section 3 we suggest the weighting of a lifetime random variable based on a quality of life index. A new 'weighted' lifetime random variable 
can already posses a more natural for degradable objects property of increasing 'mortality' rates.

\section{DEGRADATION AND MORTALITY RATE}

Let $T$ denote the lifetime, described by the Cdf $F(t)$ and mortality rate $\mu(t)$. Does increasing mortality rate $\mu(t)$ really describe aging? In fact, this is a matter of a definition: in reliability theory (Barlow and Proschan, 1975), e.g., the simplest and most popular class of aging distributions is the class of distributions with increasing failure rate (IFR). The other option is the IFRA (increasing failure rate in average) when

$$
\frac{\int_{0}^{t} \mu(u) d u}{t}
$$

is increasing in $t$. Another weaker class is the class of distributions with decreasing function $m(t)$-life expectancy at age $t$, which is defined via $\mu(t)$ as:

$$
m(t)=\int_{0}^{\infty} \exp \left(-\int_{t}^{x+t} \mu(u) d u\right) d x .
$$

It follows from (1), that $m(t)$ is decreasing when $\mu(u)$ is increasing. The inverse is generally not true (Finkelstein, 2002). Therefore, the decreasing in $t$ life expectancy at age $t$ may be, in fact, a better characteristic of aging.

The foregoing expresses a statistical (black box) point of view, when the only information at hand is the mortality data. When we speak about biological aging, an underlying biological process (processes) of aging should be taken into account. Most researchers agree that aging can be described by accumulation of some kind of damage which leads to "age-related changes in an organism that adversely affect its vitality and functions", and in the current note we shall follow this interpretation.

Does damage accumulation lead to increasing mortality rates? General progressive models (Aalen and Geising, 2001) described by monotonically increasing stochastic processes of wear or degradation, often result in increasing mortality rates. But this is not a rule. The following generalization of the Strehler-Mildvan (1960) model shows that a decreasing shape of the mortality rate is also possible.

Consider a univariate first passage-type model with killing events (Singpurwalla, 1995; Aven and Jensen, 1999; Finkelstein 2003a): Let $D_{t}, t \geq 0$ denote an increasing 
stochastic process of damage accumulation (e.g. the gamma process) and $B(t)$ be a function that defines a corresponding boundary. A death (failure) occurs when $D_{t}$ exceeds $B(t)$ for the first time. Let $d(t)$ denote the increasing sample path of this process.

Assume that $P_{t}, t \geq 0$ is an orderly (without multiple occurrences) point process of external harmful events (external stresses) with rate $\lambda(t)$. Assume also that this process is independent from $D_{t}, t \geq 0$. Following reliability terminology, we will call these events for convenience "shocks". Let $N(a, b)$ denote the number of shocks in $[a, b)$. Each shock, independently from the previous ones, results in death with probability $\theta(t)$ and is 'survived' with the complementary probability $1-\theta$. This can be interpreted in the following way: each shock has a random magnitude $Y_{i}=Y, i=1,2, \ldots$ with a distribution function $\Psi(y)$. The death at age $t$ occurs when this magnitude exceeds the margin: $B(t)-d(t)$. Therefore:

$$
\theta(t)=\operatorname{Pr}(Y>B(t)-d(t))=1-\Psi(B(t)-d(t)) .
$$

In the original Strehler-Mildvan (1960) model, which was widely applied to human mortality data, our $B(t)-d(t)$ has a meaning of vitality. It was also supposed that this function linearly decreases with age and that the distribution function $\Psi(y)$ is exponential (Yashin et al, 2000). We do not need these stringent assumptions for the forthcoming considerations.

It is worth noting that the rate (intensity) $\lambda(t)$ does not define an arbitrary point process. However, it can be defined via its complete intensity function (Cox and Isham, 1980):

$$
\lambda\left(t ; H_{t}\right)=\lim _{\Delta t \rightarrow 0} \frac{\operatorname{Pr}\left\{N(t, t+\Delta t)=1 \mid H_{t}\right\}}{\Delta t}
$$

where $H_{t}$ specifies the point process up to time $t$ (history). For orderly point processes $\lambda\left(t ; H_{t}\right) d t$ can be interpreted as a probability of a shock occurrence in $[t, t+d t)$, given the process history up to $t$. Therefore, the conditional mortality rate is:

$$
\mu_{c}\left(t, H_{t}\right) d t=\operatorname{Pr}\left\{T \in[t, t+d t) \mid H_{t}, T\left(H_{t}\right) \geq t\right\}=\theta(t) \lambda\left(t, H_{t}\right) d t
$$


where condition $T\left(H_{t}\right) \geq t$ means that all shocks in $[0, t)$ were survived (for the specific configuration of shocks given by the history $H_{t}$ ). It is important to keep in view that conditional mortality rate is based on the internal 'individual' information and in general bears no usual exponential relationship with the corresponding survival function: $\bar{F}(t) \equiv 1-F(t)=\operatorname{Pr}(T>t)$. However, only for the specific case of the Poisson process (unfortunately Strehler-Mildwan (1960) did not make this crucial assumption) equation (2) reduces to the conventional, not history-dependent mortality rate $\mu(t)$,

$$
\mu_{c}\left(t, H_{t}\right)=\theta(t) \lambda(t)=\mu(t) .
$$

Therefore, the conventional exponential representation for the corresponding survival function is

$$
\bar{F}(t)=\exp \left\{-\int_{0}^{t} \theta(u) \lambda(u) d u\right\}
$$

and this completes the proof for the specific case of the Poisson process of shocks.

Coming back to our setting, equation (4) can be obviously modified to:

$$
\bar{F}(t)=\left\{\begin{array}{lr}
\exp \left\{-\int_{0}^{t} \theta(u) \lambda(u) d u\right\}, & t \leq t_{r}, \\
0, & t>t_{r}
\end{array}\right.
$$

where $t_{r}$ is defined as the minimal solution of equation $B(t)=d(t)$. If the curves $B(t)$ and $d(t)$ does not cross, then $t_{r}=\infty$. Humans and other organisms do not usually dye directly from accumulated damage, which is a slowly increasing process. Therefore, we can assume that formally $t_{r}=\infty$ and relations (3) and (4) hold.

We have derived equations (2) - (4) for the sample path $d(t)$. A general case of the process $D_{t}, t \geq 0$ can be considered by obtaining expectation of the right hand side of (3) with respect to $D_{t}, t \geq 0$, whereas the mortality rate in this case is given by obtaining conditional expectation of the right hand side of (4) (Yashin and Manton, 1997; Finkelstein 2003a). This conditioning can only result in additional deceleration or decrease of the observed mortality rate.

Equation (3) states that the resulting mortality rate is just a simple product of the rate of the Poisson process and of the probability $\theta(t)$. Therefore, its shape can be easily analyzed. When $B(t)-d(t)$ is decreasing, the probability of death $\theta(t)$ is in- 
creasing with age, which goes in line with the accumulation of degradation reasoning. If, additionally, the rate of harmful events $\lambda(t)$ is not decreasing, or decreasing not faster than $\theta(t)$ is increasing, the resulting mortality rate $\mu(t)$ is also increasing. In conventional settings $B(t)$ is usually assumed to be a constant, therefore $B(t)-d(t)$ is decreasing automatically. On the other hand, the following properties and their obvious combinations can result in the decreasing mortality rate $\mu(t)$ :

a. $\theta(t)$ is decreasing, as the boundary function $B(t)$ is increasing faster than $d(t)$ : additional vitality is additively 'earned' by an organism with age (some relevant general models for this case can be considered, which is a topic for a special study).

b. $\theta(t)$ is decreasing, as the magnitude of harmful events $Y_{t}$ is stochastically decreasing with age $t$.

c. $\theta(t)$ is decreasing, as a resistance (or defense) of an organism to shocks is increasing with age. (It is not clear how this property go together with the general decline of vitality and functions, but probably some explanation can be found).

c. The rate of harmful events $\lambda(t)$ is decreasing. This assumption can be quite realistic, e.g., for human populations in developed countries when the exposure to stresses of different kinds decreases at advanced old ages.

Thus the case of negative aging can still occur within the framework of the suggested generalized Streller-Mildwan model. This supports our claim that in general the shape of mortality rate alone is not sufficient for defining aging properties, whereas the accumulated damage, which is responsible for age related changes in an organism, combined with other factors, eventually determines the shape of the mortality rate. On the other hand, it seems intuitively unnatural that a degradable object is characterized by the decreasing mortality rate. Therefore, in the next section a regularization procedure will be suggested which can eventually boil down in the increasing 'mortality' rate for a supplementary lifetime random variable.

\section{QUALITY OF LIFE FUNCTION}


Denote by $q(t) \leq 1$-a quality of life index at age $t$. In fact, this should be an individual stochastic function of degradation $q(D(t)))$, but we restrict ourselves to some general averaged characteristic. The function $q(t)$ defines a weight which is given to a unit increment of life at age $t$. As it was stated in the Introduction, humans at advanced ages usually have restrictions of various kinds, showing a substantial decrease in vitality and functions, which decrease a quality of life at this stage. Although formally vitality and functions decrease at all adult ages, the noticeable decline in the corresponding quality of life due to these processes occurs usually only at relatively advanced ages.

These considerations are somehow similar to the starting point of the Quality Adjusted Life Years (QALYs) approach (see, e.g., Humnik et al, 2001), but the goal is different. This approach is focused on solving individual health care decision problems, when, for instance, an operation with probability $p$ can add a number of quality years $(q=1)$, but can result in death $(q=0)$ with probability $1-p$. Without an operation a patient lives with a lower quality of life: $q<1$. Our interest is not in a specific decrease in abilities of individuals with concrete health problems, but rather in modeling a general population trend, which shows the decline in quality of life as the manifestation of senescence. Therefore, we will assume that $q(t)=1, t \in\left[0, t_{s}\right)$ and that this function monotonically decreases for $t \geq t_{s}$, where $t_{s}$ is the starting point of senescence: a noticeable decline in 'abilities and possibilities'.

Consider the remaining lifetime at some $x \geq t_{s}$, Denote this random variable by $T_{x}$ and the corresponding Cdf by $F(t-x \mid x), t \geq x$. Thus:

$$
\begin{aligned}
& 1-F(t-x \mid x)=\frac{1-F(t)}{1-F(x)} \\
& =\exp \left\{-\int_{x}^{t} \mu(u) d u\right\}=\exp \left\{-\int_{0}^{t-x} \mu(x+u) d u\right\} .
\end{aligned}
$$

Denote by $T_{q}$ a 'weighted lifetime': random variable weighted in accordance with the quality of life function $q(t)$ :

$$
T_{q}=\int_{0}^{T} q(u) d u \equiv Q(T),
$$

where $Q^{\prime}(t)=q(t)$. We must also assume for simplicity that 


$$
\lim _{t \rightarrow \infty} Q(t)=\infty
$$

although the situation for a non-proper case, when this limit is less than $\infty$, can be also considered.

It is clear that, when $q(t) \equiv 1$, the lifetimes are equal: $T_{q}=T$. Thus, $T_{q}$ already reflects in a certain way not only the length of life but its quality as well. The corresponding distribution function is easily derived:

$$
G(t)=\operatorname{Pr}(Q(T) \leq t)=\operatorname{Pr}\left(T \leq Q^{-1}(t)\right)=F\left(Q^{-1}(t)\right),
$$

where $Q^{-1}(t)$ is the inverse function to $Q(t)$, which exists and increases due to definition (5). Relation (6) defines the accelerated life model (Finkelstein, 1999) with a scale transformation depending on age $t$.

It can be shown that the 'mortality rate' defined by the distribution $G(t)$ is:

$$
\mu_{q}(t)=\frac{d\left(Q^{-1}(t)\right)}{d t} \mu\left(Q^{-1}(t)\right)
$$

Our intention is to show that, for instance, in cases of ultimately decreasing mortality rate $\mu(t)$, which are usually qualified as negative senescence, the function $\mu_{q}(t)$ can increase, which is somehow more intuitively acceptable for models with degradation. It is natural to model $q(t)$ as a decreasing power function for large $t$. A generalization to the regularly varying functions (Bingham et al, 1987) is rather straightforward. Let: $q(t) \sim t^{-\alpha}, 0<\alpha<1$. By this notation we mean proportionality. The case: $\alpha=1$ will be considered separately, whereas the range $\alpha>1$ is not allowed by relation (6). Then:

$$
Q(t) \sim t^{-\alpha+1}=t^{\frac{k}{n}} ; k<n, \quad Q^{-1}(t) \sim t^{\frac{n}{k}} .
$$

Therefore, as follows from definition (7), e.g, for a constant mortality rate $\mu(t)$, the rate $\mu_{q}$ is already increasing and $\mu_{q}(t) \sim t^{\frac{n}{k}-1}$. It is easy to see that it will be still increasing even for decreasing mortality rates: $\mu(t) \sim t^{-B}$, if $0<\beta<1-\frac{k}{n}$. Thus, under some reasonable assumptions a 'regularization' procedure had been performed resulting in the increasing rate $\mu_{q}(t)$. The following example deals with the case: $\alpha=1$. 
Example. Let $F(t)=1-\exp \{-\mu t\}$ and :

$$
q(t)=\left\{\begin{array}{lr}
1, & t \leq t_{s} \\
\frac{k}{\left(t-t_{s}\right)+k}, & t>t_{s}
\end{array}\right.
$$

where $k>0$, which means that for sufficiently large $t: q(t) \sim k / t$. Then:

$$
Q(t)= \begin{cases}t, & t \leq t_{s} \\ t_{s}+k\left[\ln \left(\frac{t-t_{s}}{k}+1\right)\right], & t>t_{s}\end{cases}
$$

It is easy to see that the inverse function $Q^{-1}(t)$ is linear in $\left[0, t_{s}\right]$ and is exponentially increasing for $t>t_{s}$. It follows from equations (7) and (8) that $\mu_{q}(t)$ is also increasing for $t>t_{s}$ ( exponentially) and is constant in $\left[0, t_{s}\right]$. This shape already reflects degradation in the model. The same, in accordance with relation (7), is true for the case when $\mu(t)$ is decreasing but slower than $\left(Q^{-1}(t)\right)^{\prime}$ is increasing.

The quality of life approach is probably more natural to be used for considering the corresponding life expectancy at time $t$ than for mortality rate itself. Similar to definition (1)

$$
m_{q}(t)=\int_{0}^{\infty} \exp \left(-\int_{t}^{x+t} \mu_{q}(u) d u\right) d x
$$

which means that $m_{q}(t)$ can decrease when $m(t)$ is constant or increasing.

\section{CONCLUDING REMARKS}

Usually mortality rates increase with age as the consequence of age-related changes in an organism that adversely affect its vitality and functions. Within the framework of the generalized Strehler-Mildwan model, we show that theoretically different shapes of mortality rate functions are possible even with degradation. Modeling of probability $\theta(t)$ is crucial for this approach. The assumption that the process of shocks is the Poisson one is important for obtaining the mortality rate in the closed simple form (3). We can generalize the approach to the renewal process of shocks (Finkelstein, $2003 \mathrm{~b}$ ) and also can incorporate in the model the fact that after the successfully survived 
shock the level of accumulated damage increases on a random amount, but the corresponding technical derivations are rather cumbersome.

The suggested change of variables from $T$ to $T_{q}$, defined by relation (5), captures a natural degradation at old age, which is crudely characterized by the function $q(t)$. It should be noted, however, that from probabilistic point of view the random variable $T_{q}$ should be treated very carefully.

\section{References}

Aven, T., and Jensen, U. (1999). Stochastic Models in Reliability. Springer.

Aalen, O.O., and Gjessing, H.K. (2001). Understanding the shape of the hazard rate: a process point of view. Statistical Science, 16: 1-22.

Barlow R., and Proschan F. (1975). Statistical Theory of Reliability and Life Testing. Probability Models. New-York: Holt, Rinehart and Winston.

Bingham, N. H., Goldie, C. M., and Teugels, J. L. (1987). Regular Variation, Cambridge, University Press.

Cox D.R, Isham V. (1980). Point Processes, Chapman and Hall.

Hunink, M, Glaszion, P, Siegel, J, Piskin, J, Elstein, A, and Weinstein, M. (2001). Decision Making in Health and Medicine: Integrating Evidence and Values. Cambridge University Press.

Finch, C (1990). Longevity, Senescence, and the Genome. University of Chicago

Press, Chicago.

Finkelstein, M.S. (1999). Wearing-out components in variable environment (1999). Reliability Engineering and System Safety, 66, N3, 235-242.

Finkelstein, M. S. (2002). On the shape of the mean residual life function. Applied Stochastic Models in Business and Industry, 18: 135-146.

Finkelstein, M. S. (2003a). A model of biological aging and the shape of the observed hazard rate. Lifetime Data Analysis, 9: 93-109.

Finkelstein, M. S. (2003b). Simple bounds for terminating Poisson and renewal processes. Journal of Statistical Planning and Inference, 113, 541-548.

Singpurwalla, N. D. (1995). Survival in dynamic environment. Statistical Science, 10, 86-108. 
Strehler, B.L., and Mildvan, A. S. (1960). General theory of mortality and aging. Science, $132,14-21$.

Vaupel, J. W., Manton K. G., and Stallard E. (1979). The impact of heterogeneity in individual frailty on the dynamics of mortality. Demography, 16: 439-454.

Vaupel, J. W.,Baudisch, A, Dolling, M, Roach, D. A., and Gampe, J. (2004). The case of negative senescence. Theoretical Population Biology, 65, 339-351.

A.I. Yashin. A. I., and K.G. Manton, K. G. (1997). Effects of unobserved and partially observed covariate processes on system failure: a review of models and estimation strategies. Statistical Science, 12, 20-34.

Yashin,A. I, Iachine, I.A., and Begun, A. S. (2000). Mortality modeling: a review.

Mathematical Population Studies, 8: 305-332. 